\title{
Idiopathic hypereosinophilic syndrome presenting as childhood hemiplegia
}

\author{
K. Ashok Kumar, A. Anjaneyulu and J.M.K. Murthy
}

Department of Neurology, The Nizam's Institute of Medical Sciences, Punjagutta, Hyderabad-500 482, Andhra Pradesh, India

\begin{abstract}
Summary: A case of childhood hemiplegia due to idiopathic hypereosinophilic syndrome is reported. There was no cardiac lesion. The neurological complications associated with hypereosinophilic syndrome and the pathophysiological mechanism of neurotoxicity of human eosinophils are discussed. It is likely that the neurological deficit was due to eosinophilic neurotoxicity.
\end{abstract}

\section{Introduction}

The idiopathic hypereosinophilic syndrome (HES) represents a heterogeneous group of disorders with the common features of prolonged eosinophilia of an undetectable cause and organ system dysfunction. Diagnostic criteria have been established for the idiopathic hypereosinophilic syndrome. They include persistent eosinophilia of 1,500 eosinophils $/ \mathrm{mm}^{3}$ for at least 6 months or death before 6 months with signs and symptoms of HES, lack of evidence for other recognized causes of eosinophilia despite careful evaluation, and signs and symptoms of organ dysfunction either directly related to eosinophilia or unexplained in the given clinical setting. ${ }^{1,2}$ Neurological manifestations of HES include: (a) diffuse central nervous system abnormality characterized by altered behaviour and cognitive dysfunction, spasticity and, occasionally, ataxia: (b) peripheral neuropathy; and (c) focal central nervous system deficits from cardiac emboli or haematological disturbances. ${ }^{3-5}$ The neurological symptoms and signs can precede the first symptoms of cardiac involvement. ${ }^{3}$ This paper presents a child with idiopathic HES in whom the initial presentation was hemiplegia.

\section{Case report}

An 8 year female child was admitted with sudden onset of weakness of the left half of the body. There was no history of seizures, altered sensorium, headache or vomiting. She had a low grade fever one week before admission. There was no history

Correspondence: J.M.K. Murthy, M.D., D.M.(Neuro.), M.N.A.M.S.(Neuro.).

Accepted: 3 March 1992 suggestive of bronchial asthma, nasal allergy, skin rash or worm infestation. She was not from an endemic area for filariasis.

On examination, she was fully alert and well orientated. Speech and ocular fundi were normal. There was a left upper motor facial nerve palsy and hemiparesis, power was graded at $2-3 / 5$. The tendon reflexes were brisk on the left side with an extensor plantar response. Sensory and cerebellar systems were normal. She had mild hepatosplenomegaly. Lungs were clinically normal. There were no cardiac murmurs.

Investigations showed a total white cell count of $20,800 / \mathrm{mm}^{3}$ with $63 \%$ eosinophils. The absolute eosinophil count was $13,200 / \mathrm{mm}^{3}$. The erythrocyte sediment rate was $150 \mathrm{~mm} /$ hour. The patient was started on steroid therapy and was referred to this Institute for further management, when the absolute eosinophil count was $1,500 / \mathrm{mm}^{3}$. Bone marrow examination revealed a normal erythroidmyeloid ratio, and $10 \%$ eosinophilia without premature cells. Repeated examinations of stool were negative for any ova or cyst. Blood smears were negative for haemoparasites. Casoni and Mantoux tests were negative. Sputum was negative for acid-fast bacilli by smear and culture. Biochemical and cytological analysis of cerebrospinal fluid was normal without any eosinophilia. No micro-organisms, including Toxocara canis and Angiostrongylus cantonensis, could be isolated from it. Chest $\mathrm{X}$-ray showed evidence of miliary mottling and ultrasonic examination of the abdomen confirmed hepatosplenomegaly. The electrocardiogram and two-dimensional echocardiographic examinations were essentially normal. A computed tomography scan of the brain showed hypodense lesions bilaterally in the parietal regions (Figure 1). There was no significant enhancement on contrast. 


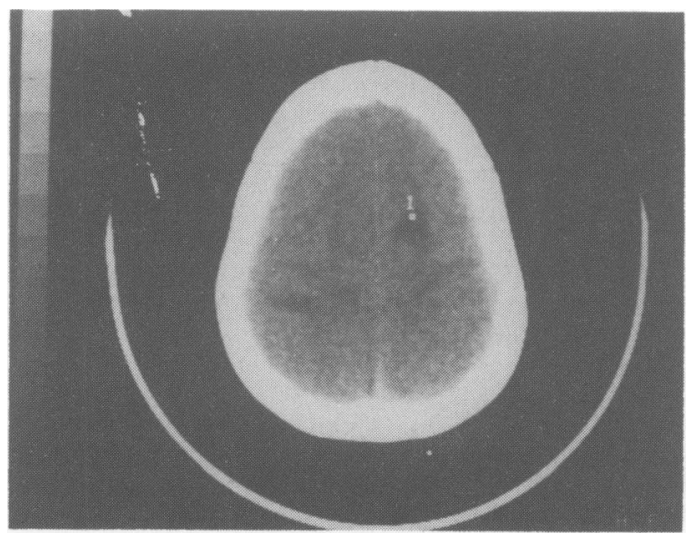

Figure 1 Computed tomographic scan of the brain showing a definite low attenuation area in the right parietal region with ill-defined low attenuated areas in both parietal lobes.

The child was restarted on prednisolone and the absolute eosinophil count was monitored during follow-up. The patient improved in the next 3 months and recovered completely from the hemiparesis. At 4 weeks, the absolute eosinophil count was $1,800 / \mathrm{mm}^{3}$ and it further fell to $200 / \mathrm{mm}^{3}$ at 6 weeks and to $100 / \mathrm{mm}^{3}$ at 3 months. Attempts to withdraw steroids completely have resulted in increasing eosinophil counts within a few weeks, necessitating a continuous maintenance dose of steroids. Repeat chest X-ray showed clearance of the miliary mottling and ultrasound examinaton of abdomen showed regression of hepatosplenomegaly. The patient is under regular follow-up and there is no evidence of other organ involvement.

\section{Discussion}

This patient had clinical and laboratory features which fulfilled the diagnostic criteria of idiopathic HES. She presented with hemiparesis accompanied by hypereosinophilia, hepatosplenomegaly and miliary mottling of the lungs. There was no evidence of worm infestation and the clinical presentation is unlike that of an unusual infection like Angiostrongylus cantonensi, since the patient had no meningitic illness or cerebrospinal fluid abnormalities. ${ }^{6,7}$ Moreover, A. cantonensi infection is

\section{References}

1. Chusid, M.J., Dale, D.C., West, B.C. \& Wolff, S.M. The hypereosinophilic syndrome. Analysis of fourteen cases with review of literature. Medicine 1975, 54: 1-27.

2. Parillo, J.E., Fauci, A.S. \& Wolff, S.M. Therapy of hypereosinophilic syndrome. Ann Intern Med 1978, 89: 167-172. rare in this country, only two cases having been $\frac{\varrho}{3}$ reported so far. ${ }^{8}$ The low bone marrow esosino- $\frac{\mathbb{\Phi}}{2}$ phila seen in this patient could be related to the $\frac{\varrho}{C}$ steroids she received prior to admission, as the peripheral blood at the time of bone marrow examination also showed a lower eosinophil count.

Neurological complications in association with $\frac{\bar{\sigma}}{\bar{t}}$ idiopathic HES have been described in three child- $\overline{\bar{D}}$. ren, ${ }^{9-11}$ all of whom had an associated endocarditis. $\stackrel{\otimes}{\alpha}$ The neurological manifestations can be due to a thromboembolic phenomenon or to a tissuedamaging potential of the eosinophils. The ability? of the eosinophils or its contents to cause tissue $\overrightarrow{\vec{\omega}}$ damage was noted as early as in 1933 by Gordon. ${ }^{11} \stackrel{\sigma}{\omega}$ A neurotoxic fraction has been isolated from ${ }_{0}^{\circ}$ human eosinophils but not from human neutro- 3 phils. ${ }^{12}$ Intracerebral and intrathecal administration of eosinophils or eosinophil-derived neurotoxin causes weakness and incoordination in experiment- $\not$ al animals. ${ }^{11-13}$ The pathological findings disclosed $\dot{\infty}$ a loss of Purkinje cells, severe axonal damage and $\stackrel{\omega}{\Delta}$ vacuolization of the white matter of the cerebellum, $\frac{\text { ? }}{\supset}$ brainstem and spinal cord. A recent study by Sunohara et $a l .{ }^{14}$ supports the possibility that a $\bigcirc$ peripheral neuropathy can be caused by agents $\frac{?}{0}$ derived from the eosinophils. It is possible that the eosinophilic major basic protein, by its endothelial $\overrightarrow{\vec{\theta}}$ damaging effect, may also contribute to tissue $₫$ damage. ${ }^{15}$ Eosinophilic cationic protein has beef ? reported to have a profound effect on the coagula tion system in vitro. ${ }^{16}$ This protein may be partially responsible for the thromboembolic phenomenon. In this patient, there was no evidence of cardiac $ٌ$ involvement, either clinically or on echocardio- $\stackrel{\square}{\AA}$ graphic evaluation. It is likely that in this patient, $\overrightarrow{\vec{P}}$ the neurological deficit is due to agents derived $\frac{3}{3}$ from eosinophils.

The prognosis in idiopathic HES depends upon the degree of involvement of vital organs. Since the $-\bar{\partial}$ ultimate prognosis is dictated by the extent of 3 . cardiac involvement, it is imperative to start aggressive therapy in patients with cardiac involve- 3 . ment before congestive heart failure develops. ${ }^{1,2,17} \delta$ Initially, therapy is started with prednisolone, which is tapered to the lowest possible dose if the disease stabilizes or improves. If progression $D$ occurs despite steroid therapy, the antimetabolite hydroxyurea may be useful. Leukapheresis may rarely be required, if eosinophilic counts are ex- $\Omega$ tremely high. ${ }^{1,2,17}$

3. Rouzand, M., Jobard, P., Dejiovanni, E., Medielsi, M., Reynaud, J. \& Leroy, J. Manifestations neurologiques, an cours d'une endocardite fibroplastique de Loeffler. Rev $\underset{\mathbb{D}}{ }$ Neurol 1972, 126: 191-215. 
4. Rouzand, M. \& Degiovanni, E. Neurological manifestations of Loeffler's fibroplastic endocarditis. In Vinken, P.J. \& Bruyn, G.W. (eds) Handbook of Neurology, vol. 38. North Holland Publishing Company, Amsterdam, New York, Oxford, 1979, pp. 193-220.

5. Moore, P.M., Harley, J.B. \& Fauci, A.S. Neurologic dysfunction in the idiopathic hyper-eosinophilic syndrome. Ann Intern Med 1985, 102: 109-114.

6. Kuberski, T., Bart, R.D., Briley, J.M. \& Rosen, L. Recovery of Angiostrongylus cantonensis from cerebrospinal fluid of a child with eosinophilic meningitis. J Clin Microbiol 1979, 9: 629-631.

7. Yii, C.Y. Clinical observation on eosinophilic meningitis and meningoencephalitis caused by Angiostrongylus cantonensis in Taiwan. Am J Trop Med Hyg 1976, 25: 233-249.

8. Purohit, A.K., Dinakar, I., Sundaram, C. \& Ratnakar, K.S. Angiostrongylus cantonensis abscess in the brain. $J$ Neurol Neurosurg Psych 1991, 54: 11, 1015-1016.

9. Yam, L.T., Li, C.Y., Necheless, T.F. \& Katyama, I. Pseudoeosinophilia, eosinophilic endocarditis and eosinophilic leukaemia. Am J Med 1972, 53: 193-202.

10. Rasche, R.F.H., Kelsch, R.D. \& Weaver, D.K. Loeffler's endocarditis in childhood. Br Heart J 1973, 35: 774-776.

11. Gordon, M.H. Remarks on Hodgkin's disease. A pathogenic agent in the glands, and its application in diagnosis. $\mathrm{Br} \mathrm{Med} \mathrm{J}$ 1933, 1: $641-647$.
12. Durack, D.T., Sumi, S.M. \& Klebanoff, S.J. Neurotoxicity of human eosinophils. Proc Natl Acad Sci (USA) 1979, 76: 1443-1447.

13. Seiler, G., Westerman, R.A. \& Wilson, J.A. The role of specific eosinophil granules in eosinophil induced experimental encephalitis. Neurology 1969, 19: 478-488.

14. Sunohara, N., Furukawa, S., Nishio, T., Mukoyama, M. \& Satoyoshi, E. Neurotoxicity of eosinophils towards peripheral nerves. J Neurol Sci 1989, 92: 1-7.

15. Wassom, D.L., Loegering, D.A., Solley, G.O. et al. Elevated serum levels of the eosinophil granule major basic protein in patient with eosinophila. J Clin Invest 1981, 67: 651-661.

16. Venge, P., Dahl, R., Hallgren, R. \& Olsson, I. Cationic proteins of human eosinophil and their role in the inflammatory reaction. In Mahmoud, A.A.F. \& Austen, K.F. (eds). The Eosinophil in Health and Disease. Grune \& Stratton, New York, 1980, pp. 131-144.

17. Fauci, A.S., Harley, J.B., Roberts, W.C., Ferrans, V.J., Gralnick, H.R. \& Bjornson, B.H. The idiopathic hypereosinophilic syndrome. Clinical, pathophysiologic and therapeutic considerations. Ann Intern Med 1982, 97: 78-92. 